\title{
Using the Drosophila Nephrocyte to Model Podocyte Function and Disease
}

\author{
Martin Helmstädter ${ }^{1,2}$, Tobias B. Huber ${ }^{3}$ and Tobias Hermle ${ }^{1,2 *}$ \\ ${ }^{1}$ Renal Division, University Medical Center Freiburg, Freiburg, Germany, ${ }^{2}$ Faculty of Medicine, University of Freiburg, \\ Freiburg, Germany, ${ }^{3}$ III. Department of Medicine, University Medical Center Hamburg-Eppendorf, Hamburg, Germany
}

OPEN ACCESS

Edited by:

Miriam Schmidts,

Radboud University Nijmegen,

Netherlands

Reviewed by:

Andrew Mallett,

Royal Brisbane and Women's

Hospital, Australia

Michal Malina,

Charles University, Czechia

Michael Peter Krahn,

University Hospital Muenster,

Germany

*Correspondence:

Tobias Hermle

tobias.hermle@uniklinik-

freiburg.de

Specialty section:

This article was submitted to

Pediatric Nephrology,

a section of the journal

Frontiers in Pediatrics

Received: 20 October 2017 Accepted: 24 November 2017 Published: 07 December 2017

Citation:

Helmstädter M, Huber TB and Hermle T (2017) Using the Drosophila Nephrocyte to Model Podocyte

Function and Disease.

Front. Pediatr. 5:262.

doi: 10.3389/fped.2017.00262
Glomerular disorders are a major cause of end-stage renal disease and effective therapies are often lacking. Nephrocytes are considered to be part of the Drosophila excretory system and form slit diaphragms across cellular membrane invaginations. Nehphrocytes have been shown to share functional, morphological, and molecular features with podocytes, which form the glomerular filter in vertebrates. Here, we report the progress and the evolving tool-set of this model system. Combining a functional, accessible slit diaphragm with the power of the genetic tool-kit in Drosophila, the nephrocyte has the potential to greatly advance our understanding of the glomerular filtration barrier in health and disease.

Keywords: nephrocyte, Drosophila, podocyte, glomerular disease, garland cell, endocytosis, renal disease

\section{INTRODUCTION}

Disorders that affect the glomerulus are the predominant cause of end-stage renal disease (ESRD) (1). Beyond renal replacement therapy, potent therapeutic options are not available for most of the disorders from this heterogeneous group. This underscores the unmet need for valid model systems that serve to understand the mechanisms of glomerular diseases.

The most established model system for the glomerulus is currently the mouse. Despite being the undeniable gold standard, this model has its inherent limitations in costs, speed, and considerations of animal welfare. Complementary systems are thus desirable. An excellent alternative can be found in the zebrafish model. But the aforementioned limitations of the mouse model are alleviated only in parts in zebrafish, while additional obstacles like the shortcomings of the morpholino technology need to be considered. Significant insights were also derived from in vitro studies, mainly by using cultured podocytes (2). However, here other significant limitations are arising. This is mainly a consequence of the complex glomerular architecture. The glomerular filter is three-layered, including the fenestrated endothelium, the glomerular basement membrane, and the podocytes that form the slit diaphragm. Podocytes are characterized by their most intricate cell shape that is paralleled only by neuronal cells. Cultured podocytes lack crucial features of their in vivo counterparts, most notably the formation of slit diaphragms. The Drosophila anatomy contains no structure whose analogy to the mammalian kidney appears obvious. Thus, it was unexpected that the Drosophila nephrocyte was identified as an invertebrate model that has the potential to meet the needs for a complementary model for glomerular disease $(3,4)$.

Discovered more than 150 years ago (5) and identified as storage kidneys, the nephrocyte received limited attention until after the discovery that their auto-cellular junctions need to be considered as slit diaphragms that are formed by the orthologs of the mammalian slit diaphragm proteins nephrin (sns) and NEPH1 (kirre) $(3,4)$. Loss-of-function of slit diaphragm proteins results in a smooth 
cell surface analogous to podocyte foot process effacement. Nephrocytes thus share molecular, ultrastructural, and functional features with podocytes $(3,4,6,7)$. This makes nephrocytes a unique model system with a functional slit diaphragm in a genetically highly tractable model organism. Now, nearly a decade after the introduction of the nephrocyte to a wider audience in the renal field, the purpose of this review is to examine the progress and the evolving tool-set of this model system for glomerular diseases that still has not reached its peak.

\section{NEPHROCYTE BIOLOGY}

Regarding the history and basic principles of nephrocyte biology, we refer to detailed previous reviews $(8-10)$ in order to focus on the more recent findings.

\section{Basic Concepts of Nephrocyte As an "Excretory" Organ}

The two fundamental functional subunits of renal organs throughout vertebrate biology are the glomeruli that produce an ultrafiltrate and the tubules that process the ultrafiltrate further and finally feed the resulting urine into the disposal system. Together, they serve to eliminate toxins and waste products and maintain water, salt, and $\mathrm{pH}$ homeostasis. Following similar principles, Drosophila nephrocytes are considered to be part of the fly excretory system. The Drosophila renal system has two functional subunits as well: the nephrocytes, regarded as analogous to the glomeruli, and the Malpighian tubules, regarded as analogous to the renal tubular system. There are two distinct nephrocyte populations: the pericardial nephrocytes along the heart tube and the garland cell nephrocytes in a garland-like ring around

A
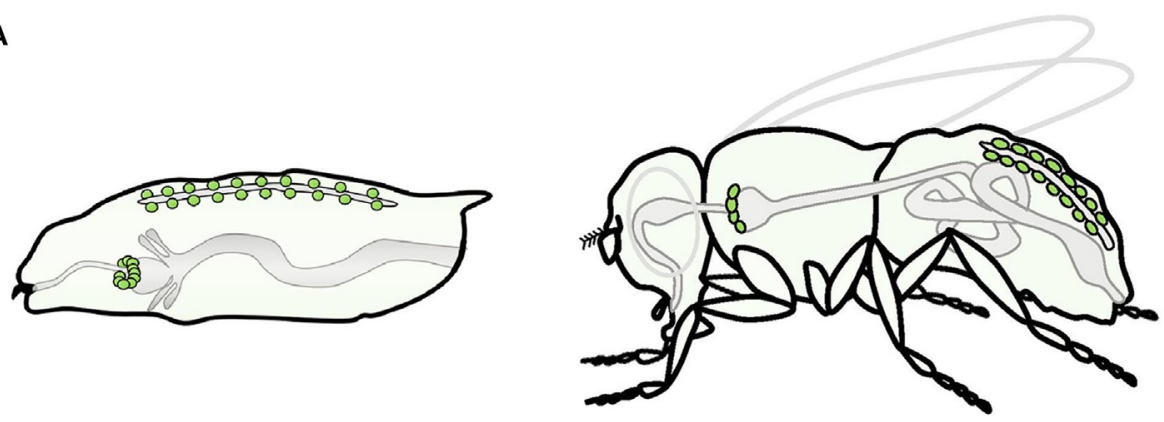

B

Mammal

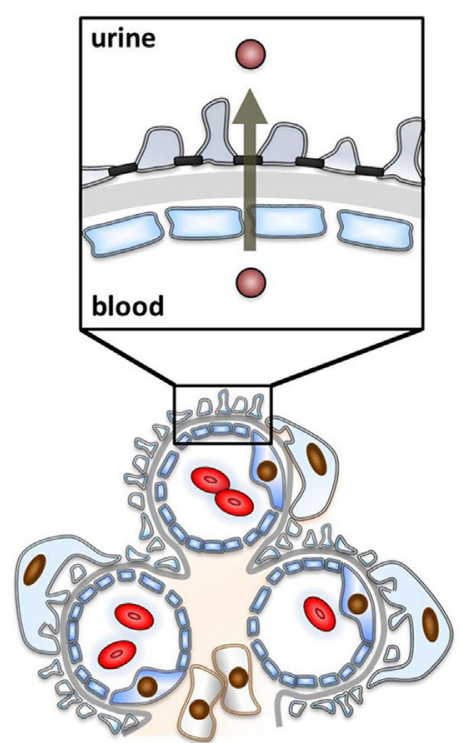

Drosophila

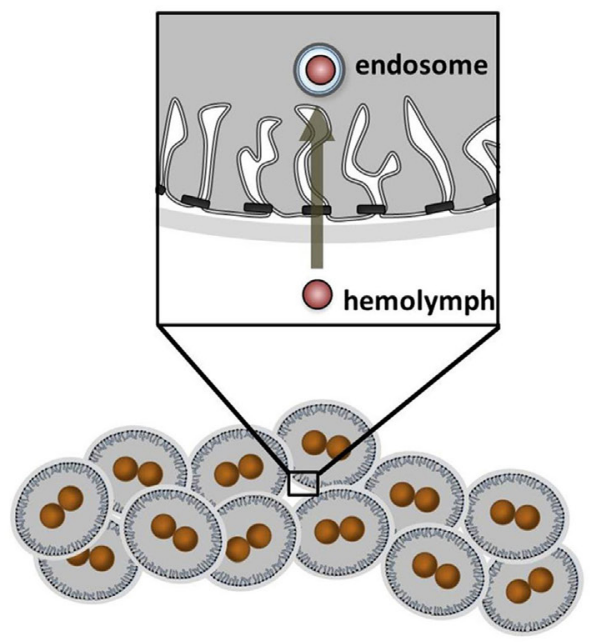

FIGURE 1 | Localization of nephrocytes and functional comparison with mammals. (A) The schematic shows the anatomical position of nephrocytes (green) within the larval body (left) and the adult fly (right). Garland cell nephrocytes localize within a ring around the esophagus and pericardial nephrocytes are found in pairs along the heart tube. (B) The schematic compares the glomerular function of mammals (left) and Drosophila (right). The mammalian glomerular filter is three-layered, consisting of the fenestrated endothelium, basement membrane, and the podocytes that form foot processes. Between these, the slit diaphragm is localized. Filtration occurs from the blood through the filter into the urinary space. In Drosophila, nephrocytes function as individual cells that are clustered. The glomerular filter is bi-layered, lacking the endothelium. Filtration occurs from the larval blood, the hemolymph, being destined for processing in endosomes. 

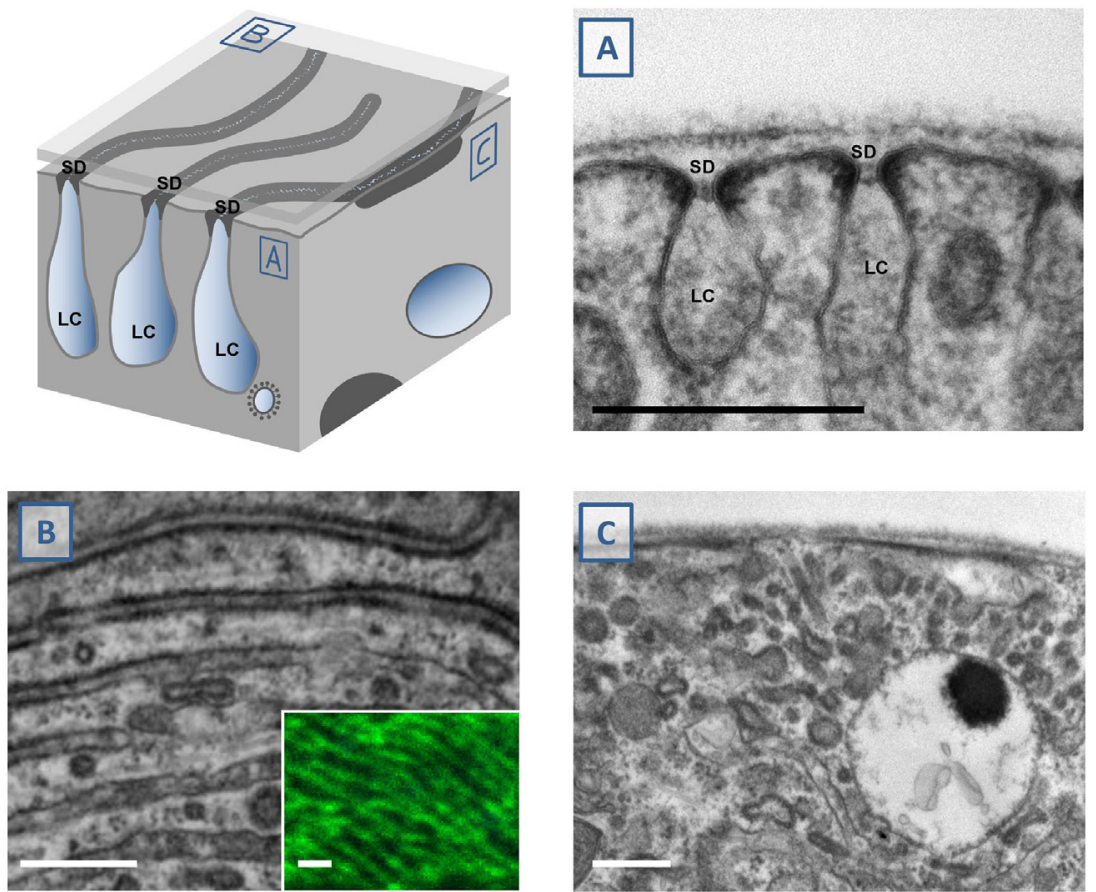

FIGURE 2 | Study of nephrocyte ultrastructural morphology using electron microscopy. The schematic illustrates a surface detail of a nephrocyte. Shown are labyrinthine channels as membrane invaginations that are sealed by the slit diaphragm. (A) The analogy to podocyte ultrastructure is most obvious in sections that are perpendicular to the nephrocyte surface. The slit diaphragm is recognizable as a fine line next to the electron-density around the entry to the membrane invagination. (B) Tangential sections through the surface show the slit diaphragm as continuous lines. This fine linear pattern correlates with the findings by confocal microscopy and staining of the ortholog of nephrin, sns (inset). (C) Perpendicular sections through the nephrocyte surface may be confusing when they cut through the nearly parallel lines of the slit diaphragms at an oblique angle. This results in an image that makes it difficult to identify the slit diaphragms and labyrinthine channels. Stretches of electron-density below the surface are mere indicators of their presence. SD indicates slit diaphragm, LC indicates labyrinthine channels, scale bars represent $500 \mathrm{~nm}$.

the esophagus (Figure 1A). However, a substantial conceptual difference to the mammalian kidney is that nephrocytes have no connection to the tubular system of Drosophila that independently generates, modifies, and finally excretes urine into the intestinal lumen (8).

\section{Brief History of Nephrocyte Research}

Despite the divergence of anatomy and functional concepts, the role of nephrocytes was already discovered in the mid-19th century. The garland cell nephrocytes were first described in 1864 by August Weismann in Freiburg. Studying larvae from Musca vomitoria, he noted a string of cells reminiscent of a garland that was floating in the larval body cavity. Accordingly, he termed them "Guirlandenzellen" (German Girlande means garland) (11). The function of these cells was an utter mystery to their discoverer but not much later, in 1886, Kowalevsky intended to stain the intestinal mucosa of muscid larvae by feeding them carmine, methylene blue or silver. However, he noted that only nephrocytes took up and stored these tracers (12). He correctly concluded from the tracer experiments that these cells function like a storage kidney to clear the larval blood. This concept was later expanded by Hollande (13). The elaborate ultrastructure of Drosophila nephrocytes was described in the sixties of the last century $(14,15)$ and few years later Crossley (16) showed the size-selectivity of uptake and already noted the analogy of this ultrastructure to podocytes: membrane invaginations called labyrinthine channels are bridged by an auto-cellular slit diaphragm that controls their entry. Nephrocytes are covered by a basement membrane. Thus, two of the three layers of the glomerular filter in vertebrates are found in Drosophila nephrocytes (Figures 1B and $\mathbf{2 A}$ ).

After the discovery of nephrin (17), Skaer (3), Abmayr and colleagues (4), finally put the pieces together and proved a molecular analogy of podocytes and nephrocytes. They noted that the orthologs are both expressed in nephrocytes and that their absence results in nephrocyte loss of the complex invaginations and a smoothing of the cell surface $(3,4)$. Interestingly, this disruption of nephrocyte ultrastructure also follows treatment with puromycine (18) and protamine sulfate (7), both of which have the same effect in podocytes. Mammalian NEPH proteins have been shown to be functional within this invertebrate system (19). A most recent and surprising discovery is that nephrocytes potentially exhibit apico-basal polarity (20). Crumbs, a marker of apical polarity, was observed along the membrane of the labyrinthine channels. The presence of integrins on the nephrocyte surface may be suggestive of a basolateral compartment (7). This raises the possibility that despite being spherical cells, nephrocytes might be polarized within 
their complex ultrastructural morphology. Consequently, analogous to mammalian podocytes, apico-basal polarity may be a potential prerequisite for nephrocyte morphology and function (20). However, at this point it has not been ruled out that the functional role of these proteins in nephrocytes is independent from polarity.

\section{Endocytosis As an Essential Nephrocyte Function}

It is astounding that nephrocytes can exert their function as an "excretory" organ without a connection to the outside world. This feat is performed by endocytosis. The first step is endocytic uptake of material from the blood of the fly that is called hemolymph. Nephrocytes then sort the endocytic cargo for either degradation in the lysosome or recycling back to the hemolymph. Unwanted cargo that cannot be destroyed, will be stored intracellularly. In that way, nephrocytes detoxify the hemolymph and nephrocyte dysfunction reduces survival upon toxin exposure (3). Nephrocytes thus do not contribute to urine formation in any way but nevertheless the high endocytic activtity can be regarded as "excretory" activity (Figure 1B). To be optimized for that purpose, they increase their surface area by forming invaginations from the cell surface called labyrinthine channels. Endocytic uptake may occur from the cell surface but the major site of endocytosis is from within the labyrinthine channels $(21,22)$. These regularly spaced furrows cover the entire nephrocyte surface $(4,7)$. The high endocytic activity attracted researchers to use nephrocytes as a model to study mechanisms of endocytosis (22-26). Endocytic uptake is at least in part dependent on the ortholog of Cubilin $(7,27,28)$. In face of this conceptual similarity to the proximal tubular function, it has been proposed that nephrocytes are also a model for the proximal tubule (28). Nephrocytes have been shown to regulate the hemolymph composition through their endocytic activity (29).

\section{Developmental Aspects}

Like podocytes, both subsets of nephrocytes are derived from the mesoderm. Differentiation and maintenance of nephrocytes requires $d K L F 15$ as a nephrocyte-restricted growth factor (30). $d K L F 15$ is the ortholog of Krüppel-Like Factor 15 which plays a role in podocyte development (31). The expression of sns and kirre and the formation of slit diaphragms begin during mid-embryogenesis and are maintained through larval development into adulthood (3, 4, 7, 32). Garland cell nephrocytes undergo a fusion process between embryonic stage E13 and E18, resulting in binucleate cells (4). For unknown reasons, the cell number decreases for both, garland cell and pericardial nephrocytes throughout development. About a quarter of the 25 garland cell nephrocytes and 120 pericardial nephrocytes that are present at the end of embryogenesis are maintained during development into adulthood $(4,7,8,33)$. Adult garland cell nephrocytes exhibit tracer uptake and show regular ultrastructural morphology, supporting these to be functional cells (7).

\section{Frontiers of Nephrocyte Research}

Despite the considerable interest nephrocytes have gained in the recent past, we do not fully understand the significance of nephrocytes within the adult fly organism. Ivy et al. (30) recorded the life-span of adult flies that lack nephrocytes due to the absence of Klf15 in comparison with wild-type. In contrast to the observations in larval stages, the absence of nephrocytes did not affect survival of adult flies, not even under toxin stress. Further work will be required to determine the functional role of nephrocytes in the organism of adult flies.

A secretory function of nephrocytes that is suggested by the abundance of rough endoplasmic reticulum has been shown for lysozyme (16) but this functional aspect has not been further explored.

Regarding the two subsets of nephrocytes, i.e., pericardial and garland cell nephrocytes, it has become overwhelmingly clear that both cells share critical features. This includes gene expression patterns of the slit diaphragm proteins and other genes like dKlf15, hand, rudhira, or Amnionless. On the other hand, obvious differences define them as distinct cell lineages, beginning with the number of nuclei. These differences are still not explored in detail. Currently, garland cell and pericardial nephrocytes are best considered to be complementary.

\section{READOUTS AND STRATEGIES OF NEPHROCYTE RESEARCH}

The functional tool-set for nephrocytes is still evolving. As a consequence, there is no defined optimal strategy or established gold standard. However, a general rule emerges that a functional and a morphological assay need to be combined. We will discuss the merits and pitfalls of different strategies to give an overview.

\section{Tracer Endocytosis As a Readout of Nephrocyte Function}

Tracer endocytosis stood at the very beginning of nephrocyte research when they enabled Kowalevski to identify clearance of the larval circulation as their functional role. Until present time, virtually any manuscript regarding nephrocytes employs tracer uptake as a functional readout. This is based on the fact that the "excretory" function of nephrocytes occurs via endocytosis. An analogous effect has also been shown for endogenous proteins like imaginal disk growth factor 2 (34).

A broad array of tracers has been used since Kowalevsky, who introduced AgNO3 that is still in use today. The most commonly used tracers are proteins like GFP-derivates (6), albumin (7), avidin (26), wheat germ agglutinin (22), or Horseradish peroxidase (HRP) (16). Another common approach uses polysaccharids like dextrans (3). Less commonly employed are colloidal substances like Coomassie Brilliant Blue (35). The choice of tracer may follow personal preference but it is important to consider the basic principles for these experiments that became clear through recent findings.

The broad range of possible tracer substances is astonishing. This reflects that the uptake of at least some tracers occurs via the scavenger receptor Cubilin (7), whose mammalian ortholog 
shows affinity for a broad range of ligands as well (36). Aside from the drastic reduction of tracer endocytosis upon silencing of Cubilin/Amnionless, this concept is supported by experiments that suggest that tracer uptake occurs receptor-mediated (7). The observation by Crossley (16) that Lanthanum-dioxide and HRP both enter the labyrinthine channels, while only the latter is taken up, indicates a certain extent of selectivity for the entry mechanism which most likely reflects the receptor affinity.

Being dependent on the scavenger receptor, measurements of nephrocyte tracer endocytosis for most tracers thus do not simply reflect the surface area of nephrocytes. The interpretation of experiments applying tracer endocytosis in nephrocytes should always take possible effects on receptor abundance, function, or specificity into account.

Filtration of tracers across the nephrocyte slit diaphragm is an important concept that directly relates to the functional analogy to podocytes and thus also underlines the significance of such experiments. Crossley (16) was able to show that in nephrocytes, tracers may be excluded size-dependently by the basement membrane and the slit membrane, thus defining a cut-off of about $12 \mathrm{~nm}$. More recently, receptor competition experiments revealed that this size cut-off is comparable with the mammalian glomerulus around $70 \mathrm{kDa}(7)$. Basement membrane thickening occurring upon loss of slit diaphragm proteins (3) mainly affects tracers with a high molecular weight while this effect is not directly related to the nephrocyte ultrastructure or filtration. This became obvious when it was shown using a rapid intervention like protamine treatment that perturbs the ultrastructure without thickening the basement membrane. This treatment has no effect on tracers with a high molecular weight while a tracer that is below the filtration cut-off is strongly reduced (7). In light of these findings, a tracer that is smaller than $70 \mathrm{kDa}$ appears to be preferable. Another potential confounding effect comes with the saturability of tracer endocytosis (7). Choosing an excessive tracer concentration or incubation period thus may even blur the effect of silencing sns despite using tracers that are small enough to pass the slit diaphragm (3).

The two major alternative strategies are a pulsed uptake by exposing nephrocytes to tracers ex vivo or using an endogenous tracer in vivo. The latter strategy was first described by Han and colleagues (6). It employs a transgenic fusion protein of red fluorescent protein with the atrial natriuretic factor from rat. This transgenic tracer is expressed endogenously by muscle cells, secreted into the larval circulation followed by endocytosis and degradation by nephrocytes. The fluorescence intensity of larval pericardial nephrocytes can be observed by transcutaneous imaging. This elegant approach is fast and enabled large-scale genetic screens (6). Studying nephrocytes in vivo, this approach appears superior to ex vivo interventions. On the other hand, one has to be conscious of certain limitations that nevertheless may favor a decision for an ex vivo setting. Imaging fluorescence through the larval cuticle may entail weak and variable signal intensity. True in vivo imaging is complicated by larval movements that usually require measures to reduce larval motility. The continuous tracer expression furthermore inherently renders the net result from uptake and degradation as readout. A block in degradation and an increase in uptake thus may have the same outcome. Possible variations in tracer production, distribution, and alternative degradation are difficult to control. The ex vivo incubation on the other hand, is able to exclusively reflect rapid uptake under controlled conditions with direct imaging of the nephrocytes. These experiments do not require the presence of a transgene that controls the expression of the endogenous tracer and thus can be immediately applied in any genetic background that allows larval survival. This approach further allows the combination with additional interventions like drug exposure. The dissection of garland cell nephrocytes can be accomplished within few seconds which minimizes the impact of an ex vivo setting. An intermediate strategy by dissecting nephrocytes from transgenic animals that express a tracer endogenously may allow to combine some of the advantages of both approaches like speed and precision of direct imaging. Then again, this also retains disadvantages from both sides like the confounders entailed by continuous tracer production and the invasiveness of dissection. In summary, application of either strategy needs to be tailored to the experimental question and the available resources.

Finally, an important consideration for any uptake experiment is that it is hard to interpret without additional morphological studies. As we can learn from loss-of-function of endosomal proteins, reduced tracer uptake may occur while the slit diaphragms are maintained $(25,26)$ (or potentially also vice versa in different settings).

\section{Electron Microscopy As a Readout of Nephrocyte Morphology}

The major advantage of nephrocytes lies in the formation of functional slit diaphragms that are easily accessible. These slit diaphragms can be observed directly by transmission electron microscopy (TEM) providing the most unequivocal evidence. That way the ultrastructural analysis is an important complement for the tracer studies whose interpretation can be more difficult but that still are indispensable as a rapid functional assay. Although the technique is well established after many decades of research, study of nephrocytes still holds challenges that entail the complex ultrastructural architecture of these cells. The labyrinthine channels are nearly parallel furrows that are observed across the whole nephrocyte surface (Figure 2). The slit diaphragms seal these invaginations like a ceiling. Tangential sections through slit diaphragms therefore impress as parallel lines $(4,7)$ (Figure 2B). The interpretation of equatorial cross sections is more difficult, as they are dependent on their orientation in regard to the lines formed by the slit diaphragm. A crosssection that cuts through the slit diaphragms at an approximately perpendicular angle renders the classical image (Figure 2A). However, if the section cuts through the parallel lines of the slit diaphragms at an angle that is too oblique (Figure $2 \mathrm{C}$ ), the slit diaphragms and labyrinthine channels will be distorted and difficult to identify. For that reason, such a section may even be mistaken for a smoothing of the nephrocyte surface. However, these oblique sections are marked by elongated stretches of electron-density (Figure 2C) that clearly distinguish them from a true phenotype. Unfortunately, examples for this error can even be found in the literature. 
Analogous to the observations regarding the mammalian slit diaphragm (37), one can assume that fixation and sample preparation may influence the resulting image.

Scanning electron microscopy (SEM) can be most useful to gather information regarding cell shape and number $(4,19)$, while this may be achieved by using approaches involving light microscopy as well. The assessment of the nephrocyte ultrastructure through this approach is complicated as the labyrinthine channels are concealed by the basement membrane. Specific protocols like protease treatments can overcome this limitation but need to be considered carefully to avoid artefact.

\section{Immunofluorescence (IF) As Complementary Strategy}

Immunofluorescence of slit diaphragm proteins facilitated the discovery of nephrocytes as a model for glomerular diseases $(3,4)$. Still, this assay is regarded a complementary strategy that was omitted in a number of manuscripts. A significant recent observation concerns highly resolved tangential confocal sections of nephrocytes stained for Sns or Kirre. These stainings reveal a fingerprint-like pattern that correlates with the picture obtained in tangential sections in TEM (Figure 2A inset) (7). This not only implies that findings obtained with TEM can be backed up by stainings of the slit diaphragm proteins but also that significant information about ultrastructural aberrations can be obtained before applying TEM. Combined with the opportunity of co-IF, this allows a more comprehensive analysis for a number of questions. IF with $3 \mathrm{D}$ reconstruction also revealed that the entire cell body is covered by slit diaphragms (7). In a similar fashion, IF can be regarded as an alternative to SEM.

It remains to be seen, if super resolution imaging using stimulated emission depletion microscopy or stochastic optical reconstruction microscopy will be able to render additional information.

\section{Readouts of Mortality}

Observing survival under the exposure to toxins like silver nitrate in larvae has been described as a possible assay that directly relates to nephrocyte function. That way it may be a suitable complement for tracer strategies that documents the effect on the larval physiology. Another assay that has been introduced more recently is to record adult life-span (38). This appears a logical addition to the spectrum of investigations. However, at this point in time, it is difficult to connect reduced adult survival with nephrocyte functions due to the findings by Hartley and colleagues (30). They did not observe an altered life-span when nephrocytes were even entirely absent entirely due to lack of $d K l f 15$. Potential confounders for fly survival can result from expression in other tissues. The commonly used GAL4-drivers to control expression of RNAi do not follow anatomic structures but are dependent on gene expression patterns. It is very difficult to rule out that temporary or very limited expression in other tissues contributes to a broad compound readout like survival. At least until the role of nephrocytes in the physiology of the adult animal is better understood, in our opinion it appears prudent to rather make use of assays that are more specifically tailored to nephrocytes.

\section{Loss-of-Function Strategies}

The virtually genome-wide availability of cost-effective, offthe-shelf RNAi libraries is a major advantage of Drosophila as a model system. Loss-of-function strategies that employ RNAi are inherently limited by potential off-targets or inefficient knockdown. This may result in contradicting results between different RNAi-transgenes. A common strategy in the Drosophila field consists in analyzing at least two independent RNAi stocks to confirm the findings. Further confirmation often remains desirable. This can be achieved by utilizing genetic null alleles. However, lethality frequently impedes this strategy like in the case of sns. Nephrocytes are not amenable to the classic mosaic analysis in Drosophila as they are non-dividing cells. The CRISPR/Cas9 technology allows to introduce a conditional loss-of-function that is not RNAi dependent (7). This can be accomplished by transgenic Drosophila that combine nephrocyte-restricted expression of Cas9 in conjunction with ubiquitous expression of a directed guide RNA. In this approach, individual cells may have divergent phenotypes dependent on individual CRISPR/ Cas-induced mutations. Possible draw backs of this strategy are off-targets, inefficient gene disruption, and persistence of mRNA. Therefore, it is reasonable to either use two independent guide RNAs or combine this with an RNAi approach. For both methods, RNAi and CRISPR/Cas, loss-of-function best is further verified, e.g., by IF using a specific antibody or rescue experiments with an RNAi/CRISPR-resistant transgene.

A number of different GAL4-driver lines have been utilized to control the expression of transgenes to induce loss-offunction in nephrocytes. These include Dorothy-GAL4 $(6,39)$, sns-GCN-GAL4 (4, 40), and Hand-GAL4 (41). While these lines express GAL4 in both subsets of nephrocytes, the equally common prospero-GAL4 $(3,42)$ has predominantly been used for garland cell nephrocytes. A garland cell nephrocyterestricted expression has been described for Aug21-GAL4 (43). All commonly used driver lines appear to exhibit robust expression during the larval stage. G447.2-GAL4 (3) was used to direct expression in embryonic garland cell nephrocytes. Dorothy-GAL4 (7, 44, 45), Hand-GAL4 (46), and sns-GCNGAL4 (47) have been employed in nephrocytes dissected from adult animals. A systematic comparison of the available GAL4 lines regarding onset, continuation, and intensity of transgene expression is currently lacking. The same is true for a characterization of the extra-nephrocytic expression that almost certainly occurs in all the GAL4-drivers.

\section{NEPHROCYTE AS DISEASE MODEL}

\section{Monogenic Forms of Nephrotic Syndrome}

Drosophila shows a surprising extent of conservation of disease genes and this model organism has proven to be appropriate for the investigation of monogenic diseases. These disorders are characterized by mutation of a single gene as the molecular cause of a disease. The role of nephrocytes as a model for genetic kidney disease has recently been reviewed in more detail (48). Nephrocytes are suitable to study the functional role of candidate genes that were identified in genetic studies and also elucidate 
the involved functional pathways which is facilitated by the low redundancy of the fly genome. The speed, cost-effectiveness, and reliability of this model make it well equipped to handle the considerable number of candidate variants that may result from contemporary sequencing efforts like whole exome sequencing. Beyond that, nephrocytes can also be applied to assess the significance of individual candidate mutations using transgenic rescue constructs. In the near future, Drosophila nephrocytes may further facilitate the development of targeted therapies and personalized medicine.

The most extensive work in Drosophila nephrocytes concerns steroid resistant nephrotic syndrome (SRNS). This disorder is characterized by childhood onset of edema, massive proteinuria, and progression to ESRD. SRNS is the second most frequent cause of ESRD within the first two decades of life and monogenic mutations explain a significant fraction of cases that manifest before 25 years of age (49). By now, more than 50 different genes have been implicated as a single gene cause for this disorder $(50,51)$ and more genes are likely to be discovered. The large number of known SRNS genes prompted systematic analyses that showed that about $60-80 \%$ of the studied orthologs of human disease genes resulted in a reduction of tracer uptake $(7,38)$ including genes that are involved in the slit diaphragm complex, actin regulation, $\mathrm{CoQ}_{10}$-biosynthesis, or interaction with the extracellular matrix. This bears testimony to the surprising extent of evolutionary conservation and underlines that central pathomechanisms of podocytopathies can be studied in nephrocytes. This is most obvious for COQ2-nephropathy (52) where findings in Drosophila indicate formation of reactive oxygen species as a critical event in the pathogenesis. Successful drug treatment of flies further provides evidence for Vanillic acid as a potential treatment (7). Importantly, these data further support that COQ2-nephropathy may be treatable (53) and that treatment strategies can be tested in nephrocytes. Some of these findings were confirmed in a recent manuscript that furthermore implicated autophagy and mitophagy as pathomechanistically relevant (54).

For the identification of several of the SRNS genes, studies in nephrocytes were instrumental by providing functional evidence supporting the genetic findings. This includes the KANK proteins (55), ARHGDIA (56), ADCK4 (57), and most extensively SGPL1 (58). For the ortholog of SGPL1, rescue constructs that reflect the mutations from SRNS patients were shown to be inefficient in contrast to a rescue with wild-type constructs. Drosophila nephrocytes also exhibited an altered lipid metabolism reflecting the observations in the SRNS patients.

\section{Diabetic Nephropathy}

Diabetic nephropathy represents the single most significant cause of ESRD (1). Targeted therapies are unavailable and the current regimes are only sufficient to slow down the progress of disease. It is thus noteworthy that nephrocytes were found to be a model for diabetic nephropathy (59). High-glucose diet was shown to induce nephrocyte dysfunction and decrease the amount of Sns, which represents the ortholog of nephrin. The authors identified a transcriptional downregulation by a pathway that includes Knot, the ortholog of EBF2, to be responsible for the reduction of $\mathrm{Sns}$ in nephrocytes. Interestingly, the authors were able to validate their findings in Drosophila nephrocytes in mouse models of diabetic nephropathy.

\section{APOL1-Associated Nephropathies}

Incidence of ESRD is nearly fourfold in African Americans compared with those of European descent. Much of the excess risk is attributable to two risk alleles of the APOL1 gene. The study of APOL1 is hampered by its poor evolutionary conservation as orthologs are lacking in most model organisms. Transgenic expression of the human APOL1 in nephrocytes has been shown to result in a cellular toxicity that was more pronounced upon expression of the human APOL1 risk alleles $(44,45)$. Nephrocyte dysfunction and loss was observed with increasing age of the flies, involving a mechanism connected to endosomal trafficking. More recently, the findings in Drosophila were confirmed by transgenic expression of the human gene in mouse (60). Essential features of the human disease were recapitulated in this model. It is intriguing, that the role of a common risk allele was studied successfully in Drosophila nephrocytes.

\section{Endocytosis and Proximal Tubular Diseases}

Imerslund-Gräsbeck syndrome is characterized by Cobalamindeficiency and tubular proteinuria while renal function is usually preserved. Mutations of Cubilin (61) and Amnionless (62) have been shown to be causative for this disorder. The orthologs of both genes are expressed in Drosophila nephrocytes, exercising an analogous role for nephrocyte function (28). Megalin, that may cause Donnai-Barrow syndrome (63) that also involves tubular proteinuria in humans, does not seem to be required for nephrocyte function $(7,27)$. A megalin-indepdendent role of Cubilin/Amnionless has also been described in humans for the absorption of vitamin $B_{12}$ in the terminal ileum (64).

\section{Organ Crosstalk}

Drosphila is an appropriate model to study communication between organs. In an effort to identify renal cardiomodulatory factors, Hartley and colleagues induced nephrocyte disruption and observed signs of a cardiomyopathy in the fly. Analyzing the hemolymph composition, they were able to identify secreted protein acidic and cysteine rich (SPARC), whose circulating levels are downregulated by nephrocytes. Reducing the gene dosage of this factor independently, through a heterozygous null allele, ameliorated the cardiomyopathy. Interestingly, SPARC has been linked to cardiac aging and metabolic syndrome in humans.

\section{Translational Applications}

The genetic tool-kit of Drosophila combined with the costeffectiveness and speed inherent to this model offer great opportunities for a translational approach. Only a glimmer of these opportunities can be recognized in the effect of Vanillic acid on 
defective $\mathrm{CoQ}_{10}$-biosynthesis (7). Screening platforms have been introduced that already enable large-scale genetic screens. These protocols need to be optimized to facilitate the discovery of treatment options for glomerular diseases.

\section{OUTLOOK}

We have seen significant progress in the last decade, and the nephrocyte currently emerges from the phase of experimental establishment to a driving force of glomerular discovery research. Combining a functional, accessible slit diaphragm with the power of the genetic tool-kit in Drosophila, the nephrocyte as a complementary model system is well equipped to reveal mechanisms of podocyte function and glomerular diseases. Once the enormous potential for translational applications is unlocked, the nephrocyte will play its role in the identification of targeted therapies that are urgently needed in nephrology.

\section{REFERENCES}

1. United States Renal Data System. 2016 USRDS Annual Data Report: Epidemiology of Kidney Disease in the United States. Bethesda, MD: National Institutes of Health, National Institute of Diabetes and Digestive and Kidney Diseases (2016).

2. Saleem MA, O'Hare MJ, Reiser J, Coward RJ, Inward CD, Farren T, et al. A conditionally immortalized human podocyte cell line demonstrating nephrin and podocin expression. J Am Soc Nephrol (2002) 13:630-8.

3. Weavers H, Prieto-Sanchez S, Grawe F, Garcia-Lopez A, Artero R, WilschBrauninger $\mathrm{M}$, et al. The insect nephrocyte is a podocyte-like cell with a filtration slit diaphragm. Nature (2009) 457:322-6. doi:10.1038/nature07526

4. Zhuang S, Shao H, Guo F, Trimble R, Pearce E, Abmayr SM. Sns and Kirre, the Drosophila orthologs of Nephrin and Neph1, direct adhesion, fusion and formation of a slit diaphragm-like structure in insect nephrocytes. Development (2009) 136:2335-44. doi:10.1242/dev.031609

5. Kowalevsky A. Ein Beitrag zur Kenntnis der Excretionsorgane. Biol Centralbl (1889) 9:74-9.

6. Zhang F, Zhao Y, Han Z. An in vivo functional analysis system for renal gene discovery in Drosophila pericardial nephrocytes. J Am Soc Nephrol (2013) 24:191-7. doi:10.1681/ASN.2012080769

7. Hermle T, Braun DA, Helmstadter M, Huber TB, Hildebrandt F. Modeling monogenic human nephrotic syndrome in the Drosophila garland cell nephrocyte. J Am Soc Nephrol (2017) 28:1521-33. doi:10.1681/ASN.2016050517

8. Denholm B, Skaer H. Bringing together components of the fly renal system. Curr Opin Genet Dev (2009) 19:526-32. doi:10.1016/j.gde.2009.08.006

9. Simons M, Huber TB. Flying podocytes. Kidney Int (2009) 75:455-7. doi:10.1038/ki.2008.653

10. Cagan RL. The Drosophila nephrocyte. Curr Opin Nephrol Hypertens (2011) 20:409-15. doi:10.1097/MNH.0b013e328347ae02

11. Weismann A. Die nachembryonale Entwicklung der Musciden nach Beobachtungen an Musca vomitoria und Sarcophaga carnaria. Zeitschrift für wissenschaftliche Zoologie (1864) 14:187-336.

12. Kowalevsky A. Zum Verhalten des Rückengefäßes und des guirlandenförmigen Zellstranges der Musciden während der Metamorphose. Biol Centralbl (1886) 6:74-9.

13. Hollande A. La cellule péricardiale des insectes. Cytologie, histochemie, role physiologique. Archiv Anat Mic (1921) 18:85-307.

14. Mills RP, King RC. The pericardial cells of Drosophila melanogaster. Q J Microsc Sci (1965) 106:261-8.

15. Aggarwal SK, King RC. The ultrastructure of the wreath cells of Drosophila melanogaster larvae. Protoplasma (1967) 63:343-52. doi:10.1007/BF01252944

16. Crossley AC. The ultrastructure and function of pericardial cells and other nephrocytes in an insect: calliphora erythrocephala. Tissue Cell (1972) 4:529-60. doi:10.1016/S0040-8166(72)80029-6

17. Kestila M, Lenkkeri U, Mannikko M, Lamerdin J, McCready P, Putaala H, et al. Positionally cloned gene for a novel glomerular protein - nephrin -

\section{AUTHOR CONTRIBUTIONS}

The manuscript was written by $\mathrm{TH}$ with help from $\mathrm{MH}, \mathrm{MH}$ designed the figures with help from $\mathrm{TH}, \mathrm{TBH}$ critically reviewed and edited the manuscript and gave relevant additional insight. The title was suggested by TBH.

\section{ACKNOWLEDGMENTS}

TH is supported by the Deutsche Forschungsgemeinschaft (DFG, HE 7456/1-1, CRC 1140/associated member) and the Faculty of Medicine of the University of Freiburg. TBH is supported by the DFG (CRC 1140, CRC 992, HU 1016/8-1), by the BMBF (01GM1518C), by the European Research Council-ERC grant 616891, and by the H2020-IMI2 consortium BEAt-DKD. We thank Maria Ericsson (Harvard Medical School Electron Microscopy Facility) for technical assistance.

is mutated in congenital nephrotic syndrome. Mol Cell (1998) 1:575-82. doi:10.1016/S1097-2765(00)80057-X

18. Tutor AS, Prieto-Sanchez S, Ruiz-Gomez M. Src64B phosphorylates Dumbfounded and regulates slit diaphragm dynamics: Drosophila as a model to study nephropathies. Development (2014) 141:367-76. doi:10.1242/ dev.099408

19. Helmstadter M, Luthy K, Godel M, Simons M, Ashish, Nihalani D, et al. Functional study of mammalian Neph proteins in Drosophila melanogaster. PLoS One (2012) 7:e40300. doi:10.1371/journal.pone.0040300

20. Hochapfel F, Denk L, Mendl G, Schulze U, Maassen C, Zaytseva Y, et al. Distinct functions of Crumbs regulating slit diaphragms and endocytosis in Drosophila nephrocytes. Cell Mol Life Sci (2017) 74(24):4573-86. doi:10.1007/ s00018-017-2593-y

21. Kosaka T, Ikeda K. Reversible blockage of membrane retrieval and endocytosis in the garland cell of the temperature-sensitive mutant of Drosophila melanogaster, shibirets1. J Cell Biol (1983) 97:499-507. doi:10.1083/jcb.97.2.499

22. Narita K, Tsuruhara T, Koenig JH, Ikeda K. Membrane pinch-off and reinsertion observed in living cells of Drosophila. J Cell Physiol (1989) 141:383-91. doi:10.1002/jcp.1041410220

23. Lloyd TE, Atkinson R, Wu MN, Zhou Y, Pennetta G, Bellen HJ. Hrs regulates endosome membrane invagination and tyrosine kinase receptor signaling in Drosophila. Cell (2002) 108:261-9. doi:10.1016/S0092-8674(02)00611-6

24. Kim S, Wairkar YP, Daniels RW, DiAntonio A. The novel endosomal membrane protein Ema interacts with the class C Vps-HOPS complex to promote endosomal maturation. J Cell Biol (2010) 188:717-34. doi:10.1083/ jcb.200911126

25. Lorincz P, Lakatos Z, Varga A, Maruzs T, Simon-Vecsei Z, Darula Z, et al. MiniCORVET is a Vps8-containing early endosomal tether in Drosophila. Elife (2016) 5:e14226. doi:10.7554/eLife.14226

26. Vijayakrishnan N, Woodruff EA III, Broadie K. Rolling blackout is required for bulk endocytosis in non-neuronal cells and neuronal synapses. J Cell Sci (2009) 122:114-25. doi:10.1242/jcs.036673

27. Gleixner EM, Canaud G, Hermle T, Guida MC, Kretz O, Helmstadter M, et al. V-ATPase/mTOR signaling regulates megalin-mediated apical endocytosis. Cell Rep (2014) 8:10-9. doi:10.1016/j.celrep.2014.05.035

28. Zhang F, Zhao Y, Chao Y, Muir K, Han Z. Cubilin and amnionless mediate protein reabsorption in Drosophila nephrocytes. J Am Soc Nephrol (2013) 24:209-16. doi:10.1681/ASN.2012080795

29. Hartley PS, Motamedchaboki K, Bodmer R, Ocorr K. SPARC-dependent cardiomyopathy in Drosophila. Circ Cardiovasc Genet (2016) 9:119-29. doi:10.1161/CIRCGENETICS.115.001254

30. Ivy JR, Drechsler M, Catterson JH, Bodmer R, Ocorr K, Paululat A, et al. Klf15 is critical for the development and differentiation of Drosophila nephrocytes. PLoS One (2015) 10:e134620. doi:10.1371/journal.pone.0134620

31. Mallipattu SK, Liu R, Zheng F, Narla G, Maayan A, Dikman S, et al. Kruppellike factor 15 (KLF15) is a key regulator of podocyte differentiation. J Biol Chem (2012) 287:19122-35. doi:10.1074/jbc.M112.345983 
32. Tepass U, Hartenstein V. The development of cellular junctions in the Drosophila embryo. Dev Biol (1994) 161:563-96. doi:10.1006/dbio.1994.1054

33. Sellin J, Albrecht S, Kolsch V, Paululat A. Dynamics of heart differentiation, visualized utilizing heart enhancer elements of the Drosophila melanogaster bHLH transcription factor Hand. Gene Expr Patterns (2006) 6:360-75. doi:10.1016/j.modgep.2005.09.012

34. Broz V, Kucerova L, Rouhova L, Fleischmannova J, Strnad H, Bryant PJ, et al. Drosophila imaginal disc growth factor 2 is a trophic factor involved in energy balance, detoxification, and innate immunity. Sci Rep (2017) 7:43273. doi:10.1038/srep43273

35. Das D, Aradhya R, Ashoka D, Inamdar M. Macromolecular uptake in Drosophila pericardial cells requires rudhira function. Exp Cell Res (2008) 314:1804-10. doi:10.1016/j.yexcr.2008.02.009

36. Nielsen R, Christensen EI, Birn H. Megalin and cubilin in proximal tubule protein reabsorption: from experimental models to human disease. Kidney Int (2016) 89:58-67. doi:10.1016/j.kint.2015.11.007

37. Grahammer F, Wigge C, Schell C, Kretz O, Patrakka J, Schneider S, et al. A flexible, multilayered protein scaffold maintains the slit in between glomerular podocytes. JCI Insight (2016) 1(9):e86177. doi:10.1172/jci.insight.86177

38. Fu Y, Zhu JY, Richman A, Zhao Z, Zhang F, Ray PE, et al. A Drosophila model system to assess the function of human monogenic podocyte mutations that cause nephrotic syndrome. Hum Mol Genet (2017) 26:768-80. doi:10.1093/ $\mathrm{hmg} / \mathrm{ddw} 428$

39. Kimbrell DA, Hice C, Bolduc C, Kleinhesselink K, Beckingham K. The Dorothy enhancer has Tinman binding sites and drives hopscotch-induced tumor formation. Genesis (2002) 34:23-8. doi:10.1002/gene.10134

40. Kocherlakota KS, Wu JM, McDermott J, Abmayr SM. Analysis of the cell adhesion molecule sticks-and-stones reveals multiple redundant functional domains, protein-interaction motifs and phosphorylated tyrosines that direct myoblast fusion in Drosophila melanogaster. Genetics (2008) 178:1371-83. doi:10.1534/genetics.107.083808

41. Albrecht S, Wang S, Holz A, Bergter A, Paululat A. The ADAM metalloprotease Kuzbanian is crucial for proper heart formation in Drosophila melanogaster. Mech Dev (2006) 123:372-87. doi:10.1016/j.mod.2006.03.005

42. Ohshiro T, Yagami T, Zhang C, Matsuzaki F. Role of cortical tumour-suppressor proteins in asymmetric division of Drosophila neuroblast. Nature (2000) 408:593-6. doi:10.1038/35046087

43. Andreenkova OV, Karpova EK, Menshanov PN, Rauschenbach IY. Knockdown of InR gene in ventral nephrocytes promotes resistance to toxic stress in Drosophila melanogaster females. Genetika (2015) 51:263-5. doi:10.1134/S1022795415020027

44. Kruzel-Davila E, Shemer R, Ofir A, Bavli-Kertselli I, Darlyuk-Saadon I, OrenGiladi P, et al. APOL1-mediated cell injury involves disruption of conserved trafficking processes. JAm Soc Nephrol (2017) 28:1117-30. doi:10.1681/ ASN.2016050546

45. Fu Y, Zhu JY, Richman A, Zhang Y, Xie X, Das JR, et al. APOL1-G1 in nephrocytes induces hypertrophy and accelerates cell death. J Am Soc Nephrol (2017) 28:1106-16. doi:10.1681/ASN.2016050550

46. Davis EE, Zhang Q, Liu Q, Diplas BH, Davey LM, Hartley J, et al. TTC21B contributes both causal and modifying alleles across the ciliopathy spectrum. Nat Genet (2011) 43:189-96. doi:10.1038/ng.756

47. Na J, Musselman LP, Pendse J, Baranski TJ, Bodmer R, Ocorr K, et al. A Drosophila model of high sugar diet-induced cardiomyopathy. PLoS Genet (2013) 9:e1003175. doi:10.1371/journal.pgen.1003175

48. Helmstadter M, Simons M. Using Drosophila nephrocytes in genetic kidney disease. Cell Tissue Res (2017) 369:119-26. doi:10.1007/s00441-017-2606-z

49. Sadowski CE, Lovric S, Ashraf S, Pabst WL, Gee HY, Kohl S, et al. A singlegene cause in $29.5 \%$ of cases of steroid-resistant nephrotic syndrome. J Am Soc Nephrol (2015) 26:1279-89. doi:10.1681/ASN.2014050489

50. Lovric S, Ashraf S, Tan W, Hildebrandt F. Genetic testing in steroidresistant nephrotic syndrome: when and how? Nephrol Dial Transplant (2016) 31:1802-13. doi:10.1093/ndt/gfv355
51. Bierzynska A, Soderquest K, Dean P, Colby E, Rollason R, Jones C, et al. MAGI2 mutations cause congenital nephrotic syndrome. J Am Soc Nephrol (2017) 28:1614-21. doi:10.1681/ASN.2016040387

52. Diomedi-Camassei F, Di Giandomenico S, Santorelli FM, Caridi G, Piemonte F, Montini G, et al. COQ2 nephropathy: a newly described inherited mitochondriopathy with primary renal involvement. J Am Soc Nephrol (2007) 18:2773-80. doi:10.1681/ASN.2006080833

53. Desbats MA, Lunardi G, Doimo M, Trevisson E, Salviati L. Genetic bases and clinical manifestations of coenzyme Q10 (CoQ 10) deficiency. J Inherit Metab Dis (2015) 38:145-56. doi:10.1007/s10545-014-9749-9

54. Zhu JY, Fu Y, Richman A, Zhao Z, Ray PE, Han Z. A personalized model of COQ2 nephropathy rescued by the wild-type COQ2 allele or dietary coenzyme Q10 supplementation. JAm Soc Nephrol (2017) 28:2607-17. doi:10.1681/ASN.2016060626

55. Gee HY, Zhang F, Ashraf S, Kohl S, Sadowski CE, Vega-Warner V, et al. KANK deficiency leads to podocyte dysfunction and nephrotic syndrome. JClin Invest (2015) 125:2375-84. doi:10.1172/JCI79504

56. Gee HY, Saisawat P, Ashraf S, Hurd TW, Vega-Warner V, Fang H, et al. ARHGDIA mutations cause nephrotic syndrome via defective RHO GTPase signaling. J Clin Invest (2013) 123:3243-53. doi:10.1172/JCI69134

57. Ashraf S, Gee HY, Woerner S, Xie LX, Vega-Warner V, Lovric S, et al. ADCK4 mutations promote steroid-resistant nephrotic syndrome through CoQ10 biosynthesis disruption. JClin Invest (2013) 123:5179-89. doi:10.1172/ JCI69000

58. Lovric S, Goncalves S, Gee HY, Oskouian B, Srinivas H, Choi WI, et al. Mutations in sphingosine-1-phosphate lyase cause nephrosis with ichthyosis and adrenal insufficiency. J Clin Invest (2017) 127:912-28. doi:10.1172/JCI89626

59. Na J, Sweetwyne MT, Park AS, Susztak K, Cagan RL. Diet-induced podocyte dysfunction in Drosophila and mammals. Cell Rep (2015) 12:636-47. doi:10.1016/j.celrep.2015.06.056

60. Beckerman P, Bi-Karchin J, Park AS, Qiu C, Dummer PD, Soomro I, et al. Transgenic expression of human APOL1 risk variants in podocytes induces kidney disease in mice. Nat Med (2017) 23:429-38. doi:10.1038/ nm. 4287

61. Aminoff M, Carter JE, Chadwick RB, Johnson C, Grasbeck R, Abdelaal MA, et al. Mutations in CUBN, encoding the intrinsic factor-vitamin B12 receptor, cubilin, cause hereditary megaloblastic anaemia 1. Nat Genet (1999) 21:309-13. doi:10.1038/6831

62. Tanner SM, Aminoff M, Wright FA, Liyanarachchi S, Kuronen M, Saarinen A, et al. Amnionless, essential for mouse gastrulation, is mutated in recessive hereditary megaloblastic anemia. Nat Genet (2003) 33:426-9. doi:10.1038/ ng1098

63. Kantarci S, Al-Gazali L, Hill RS, Donnai D, Black GC, Bieth E, et al. Mutations in LRP2, which encodes the multiligand receptor megalin, cause DonnaiBarrow and facio-oculo-acoustico-renal syndromes. Nat Genet (2007) 39:957-9. doi:10.1038/ng2063

64. Jensen LL, Andersen RK, Hager H, Madsen M. Lack of megalin expression in adult human terminal ileum suggests megalin-independent cubilin/amnionless activity during vitamin B12 absorption. Physiol Rep (2014) 2(7):e12086. doi:10.14814/phy2.12086

Conflict of Interest Statement: The authors declare that the research was conducted in the absence of any commercial or financial relationships that could be construed as a potential conflict of interest.

Copyright (c) 2017 Helmstädter, Huber and Hermle. This is an open-access article distributed under the terms of the Creative Commons Attribution License (CC BY). The use, distribution or reproduction in other forums is permitted, provided the original author(s) or licensor are credited and that the original publication in this journal is cited, in accordance with accepted academic practice. No use, distribution or reproduction is permitted which does not comply with these terms. 